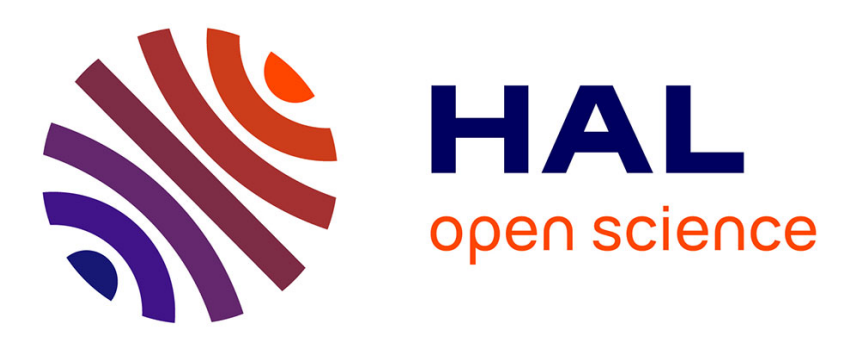

\title{
Influence of gaze elevation on estimating the possibility of passing under high obstacles during body tilt
}

Aurore Bourrelly, Lionel Bringoux, Jean-Louis Vercher

\section{To cite this version:}

Aurore Bourrelly, Lionel Bringoux, Jean-Louis Vercher. Influence of gaze elevation on estimating the possibility of passing under high obstacles during body tilt. Experimental Brain Research, 2009, 193 (1), pp.19-28. 10.1007/s00221-008-1589-0 . hal-01436029

\section{HAL Id: hal-01436029 \\ https://hal.science/hal-01436029}

Submitted on 2 May 2018

HAL is a multi-disciplinary open access archive for the deposit and dissemination of scientific research documents, whether they are published or not. The documents may come from teaching and research institutions in France or abroad, or from public or private research centers.
L'archive ouverte pluridisciplinaire HAL, est destinée au dépôt et à la diffusion de documents scientifiques de niveau recherche, publiés ou non, émanant des établissements d'enseignement et de recherche français ou étrangers, des laboratoires publics ou privés. 


\title{
Influence of gaze elevation on estimating the possibility of passing under high obstacles during body tilt
}

\author{
Aurore Bourrelly $\cdot$ Lionel Bringoux $•$ \\ Jean-Louis Vercher
}

Received: 1 April 2008/Accepted: 23 September 2008/Published online: 17 October 2008

(C) Springer-Verlag 2008

\begin{abstract}
We investigated the influence of gaze elevation on judging the possibility of passing under high obstacles during pitch body tilts, while stationary, in absence of allocentric cues. Specifically, we aimed at studying the influence of egocentric references upon geocentric judgements. Seated subjects, orientated at various body orientations, were asked to perceptually estimate the possibility of passing under a projected horizontal line while keeping their gaze on a fixation target and imagining a horizontal body displacement. The results showed a global overestimation of the possibility of passing under the line, and confirmed the influence of body orientation reported by Bringoux et al. (Exp Brain Res 185(4):673-680, 2008). More strikingly, a linear influence of gaze elevation was found on perceptual estimates. Precisely, downward eye elevation yielded increased overestimations, and conversely upward gaze elevation yielded decreased overestimations. Furthermore, body and gaze orientation effects were independent and combined additively to yield a global egocentric influence with a weight of 45 and 54\%, respectively. Overall, our data suggest that multiple egocentric references can jointly affect the estimated possibility of passing under high obstacles. These results are discussed in terms of "interpenetrability" between geocentric and egocentric reference frames and clearly demonstrate that gaze
\end{abstract}

\footnotetext{
A. Bourrelly $(\bowtie) \cdot$ L. Bringoux $\cdot$ J.-L. Vercher Institute of Movement Sciences "Etienne-Jules Marey", UMR 6233 CNRS and Université de la Méditerranée, 163, avenue de Luminy CP 910,

13288 Marseille Cedex 9, France

e-mail: aurore.bourrelly@etumel.univmed.fr

L. Bringoux

e-mail: lionel.bringoux@univmed.fr
}

elevation is involved, as body orientation, in geocentric spatial localization.

Keywords Spatial localization - Reference frames . Gaze elevation - Body orientation · Egocentric . Geocentric

\section{Introduction}

Imagine you are visiting an old castle, walking towards an open door which seems rather low. You will doubtlessly ask yourself whether you are able to pass under or not without bending. Now, imagine that before you cross the door, you pay attention to an object located on the ground, or conversely above the top of the door; will your estimation of the possibility of passing under be the same for these different gaze elevations? This study deals with the influence of gaze elevation on the estimated possibility of passing under high obstacles with different body tilts.

Motor behaviour when crossing high obstacles has already been investigated, through a task in which observers walked and passed under a horizontal barrier (Van der Meer 1997). According to Gibson's theory of affordances (1979) which claims that the environment offers a collection of possibilities for action that organisms need to detect, Van der Meer (1997) found a body-scaled critical point at which subjects began to duck under a barrier. Specifically, their results showed that subjects used a non-negligible safety margin in presence of full visual reference (for instance, a $5.25 \mathrm{~cm}$ safe margin was observed for a subject whose height is $1.75 \mathrm{~m}$ ). This cautious behaviour was also observed in full light when erect subjects had to estimate while stationary the possibility of walking under a barrier, but was not present when subjects 
sat on the floor (Wagman and Malek 2008). It was therefore suggested that intrinsic individual characteristics (body size, speed of locomotion and level of motor control) and the position of the point of observation may constitute important properties of the actor-environment fit. Other studies have demonstrated that judgements of "passability" through apertures (Mark 1987; Warren and Whang 1987) and "climbability" of objects (Warren 1984) were based on affordances for which body-scaled information were also important. As a main reference for this body-scaled information, the observers' eye level appeared determinant in estimating these possibilities of action (Marcilly and Luyat 2008; Mark 1987; Wagman and Malek 2008).

Nevertheless, although processes ruling the possibility of passing under obstacles in full vision may radically differ from those involved in complete darkness, Bringoux et al. (2008) showed that estimating the possibility of passing under a horizontal line in absence of motion at various body tilts was also referred to the estimated eye level. Interestingly, in their study, direct eye level estimates (i.e. judgements of the subjective visual horizon, that is the plane normal to gravity crossing eye level), clearly distinguished from estimates of subjective "passability". Both judgements were indeed separated by the distance between the top of the head and the physical eye level, although the slight modifications of this distance at different body orientations have not been shown to be integrated in the subjective estimates. Moreover, Bringoux et al. (2008) found a similar effect of body orientation on the subjective estimation of the height of an obstacle with respect to eye level and the possibility of passing under. Specifically, the more the subjects were tilted forward, the more they overestimated the possibility of passing under the projected line. These findings questioned the role of the vestibular system regarding the accuracy of perception of gravityspecified axes. Bringoux et al. (2007) found a similar performance in both labyrinthine-defective subjects and control subjects when judging the visual horizon in static conditions. They concluded that somatosensory inputs can convey as much graviceptive information as the vestibular system for visual horizon estimates. The observed linear relation between body orientation and the estimated possibility of passing under high obstacles was explained in terms of egocentric attraction induced by body tilt. Mittelstaedt (1983, 1986) has already explained similar phenomena by the existence of an idiotropic vector which "attracts" judgements of verticality along the longitudinal body axis. It has since been suggested that different body parts might be involved in the elaboration of the idiotropic vector (Ito and Gresty 1997). For instance, the head axis (Guerraz et al. 1998) as well as other body segments (Ito and Gresty 1997) could be involved in the egocentric influences reported in verticality judgements.
Another possible source of egocentric "attraction" may be the plane of regard. The plane of regard, containing the interocular axis and the line of sight, has been considered an important reference in egocentric spatial localization (Poljac et al. 2005; Poljac and van den Berg 2005). Specifically, the elevation of objects relative to this plane is perceived accurately, irrespective of eye or head orientation. However, the question remains whether the orientation of the plane of regard, that is, gaze elevation, is also crucial in judging the location of objects relative to some geocentric (i.e. earth-based) systems of coordinates, including the direction of gravity and the physical horizon (Howard 1982). Gaze elevation may have an influence on geocentric estimates because information about the position of the target on the retina and information about the position of the eye in the head are required for such perceptual tasks (Matin and Li 1992; Stoper and Cohen 1989). By successive transformations of coordinates, a stable map can be maintained between spatial localization, spatial orientation, and physical space (Matin and Li 1995).

The following experiment aimed first at investigating the influence of gaze elevation on estimating the possibility of passing under high obstacles during whole-body tilt, with subjects motionless, and in the absence of visual allocentric cues. The second purpose of this work was to question the relation between the influence of gaze elevation and body orientation on these judgments. A linear effect of gaze orientation was expected on the estimates since a linear body orientation influence has been previously observed by Bringoux et al. (2008).

\section{Methods}

\section{Subjects}

Twelve subjects (6 males and 6 females; mean age $27.5 \pm 9.8$ years) with normal or corrected to normal vision (by lens correction), participated in the experiment. They had no previous history of vestibular and neurological symptoms. All gave informed consent, in compliance with the ethical committee which regulates human experimentation in France.

\section{Apparatus}

The subjects were seated in complete darkness on a padded tilting chair, and restrained by means of a shoulder harness (Fig. 1). The head was strapped to a headrest which was adjusted so that the naso-occipital axis was orthogonal to the direction of the gravity when the chair was vertically oriented. The axis of rotation of the chair coincided with the trans-ocular axis. Thus, eye level remained at the same 
Fig. 1 Illustration of the experimental set-up. The motorized chair rotating around the subjects' trans-ocular axis could be rotated from $+10^{\circ}$ backward to $-10^{\circ}$ forward. The screen, $2.28 \mathrm{~m}$ away from the observers' eye supported five luminous targets (LEDs). The luminous horizontal line was projected from a laser beam at different elevations on the screen. Subjects had to rigorously fix the lighted target on the screen and to estimate whether they would be able to pass under the luminous horizontal line, imagining a horizontal displacement

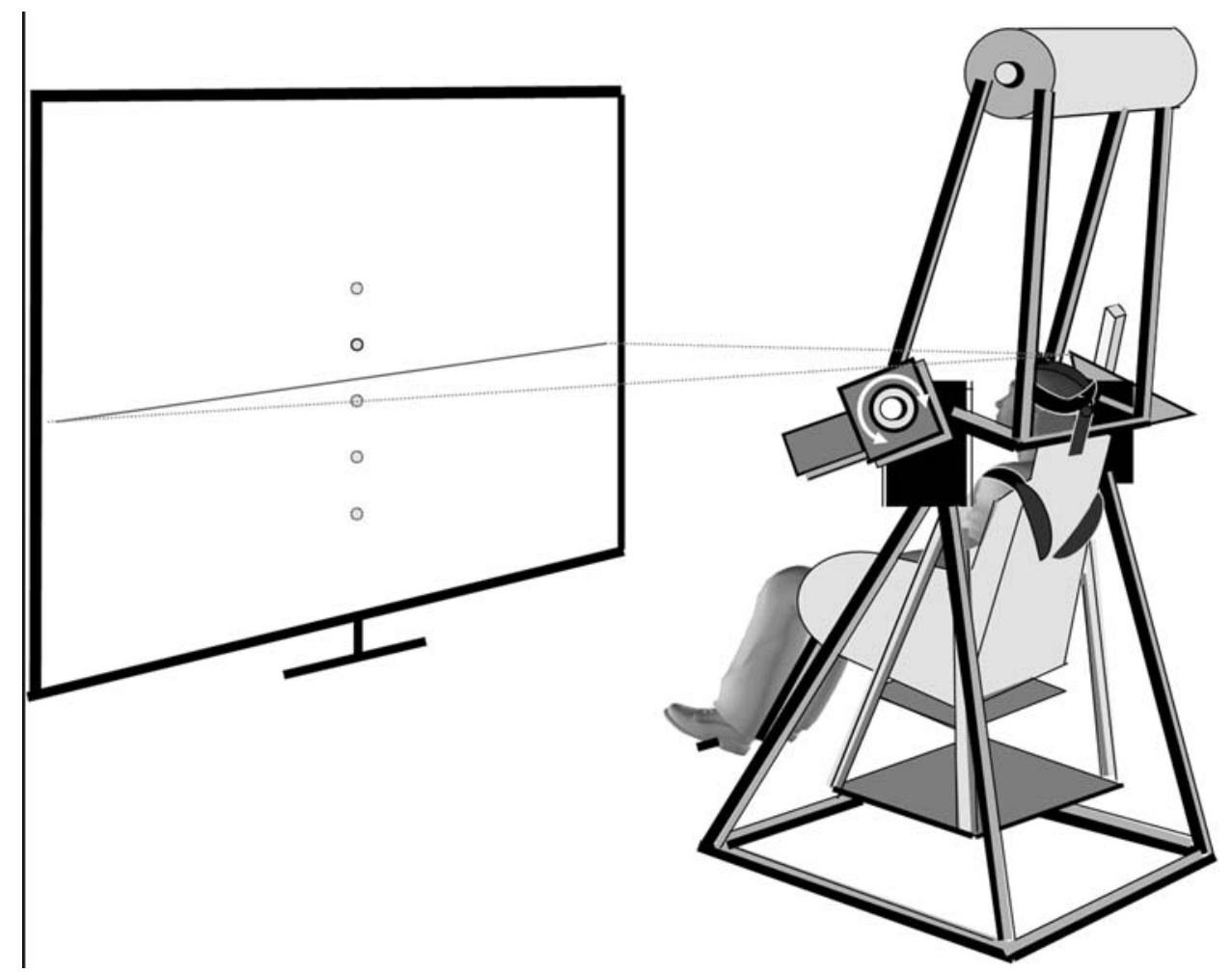

height $(1.34 \mathrm{~m})$ from the floor reference, regardless of the tilt magnitude. The motorized tilting chair, servo-controlled in speed, enabled backward and forward rotations ranging from $+10^{\circ}$ backward to $-10^{\circ}$ forward. The chair was first tilted during a $2 \mathrm{~s}$ period of initial acceleration $\left(a=0.5^{\circ} \mathrm{s}^{-2}\right)$ before reaching a constant velocity $\left(1^{\circ} \mathrm{s}^{-1}\right.$ for $-10^{\circ}$ and $+10^{\circ}$ of tilt; $v=0.375^{\circ} \mathrm{s}^{-1}$ for $-5^{\circ}$ and $+5^{\circ}$ of tilt) during $8 \mathrm{~s}$, followed by a $2 \mathrm{~s}$ period of final deceleration $\left(0.5^{\circ} \mathrm{s}^{-2}\right)$. Finally, irrespective of the angle of tilt the total duration of tilting was $12 \mathrm{~s}$.

Compliance with gaze elevation instructions was controlled online by recording subjects' eye movements (vertical DC electro-oculography, EOG). A flat vertical semi-opaque screen $2 \mathrm{~m}$ height $\times 2.5 \mathrm{~m}$ wide was placed in front of the subjects, at a distance of $2.28 \mathrm{~m}$ from the eyes. Behind the screen, five luminous targets were vertically aligned in order to define five gaze elevations $\left(+10^{\circ}\right.$, $+5^{\circ}, 0^{\circ},-5^{\circ},-10^{\circ}$ elevations from eye level). A laser pointer mounted on a fixed structure positioned beside the tilting chair projected a thin horizontal beam on a tilting mirror. The pitch orientation of the mirror was adjustable by means of a galvanometer (Scanner Control CCX 100), so that the reflected beam was projected on the screen at the desired elevation. The resulting luminous horizontal line was $2 \mathrm{~m}$ long and $0.01 \mathrm{~m}$ thick and adjustable in height with a precision of $0.01 \mathrm{~m}$. Subjects held in both hands the digital response push buttons for judgement settings. Galvanometer control and response recordings were performed by the ADwin-Gold system (Keithley ${ }^{\odot}$ ) piloted via our in-house Docometer software. Throughout the experiment, subjects were placed in darkness without any allocentric cue to influence their judgement.

\section{Procedure}

Five body orientations $\left(+10^{\circ},+5^{\circ}, 0^{\circ},-5^{\circ},-10^{\circ}\right.$, respectively, backward and forward), five gaze elevations $\left(+10^{\circ},+5^{\circ}, 0^{\circ},-5^{\circ},-10^{\circ}\right.$ elevations from eye level $)$ and ten line elevations $(+25,+20,+15,+10,+5,-5,-10$, $-15,-20,-25 \mathrm{~cm}$ from eye level; i.e., respectively, $+1.3^{\circ},+2.5^{\circ},+3.8^{\circ},+5.0^{\circ},+6.3^{\circ},-6.3^{\circ},-5.0^{\circ},-3.8^{\circ}$, $-2.5^{\circ},-1.3^{\circ}$, elevations from eye level) were manipulated in a counterbalanced pseudo-random order to prevent the possibility of any order effect. Subjects were neither informed about the number and angular values of body and gaze orientations nor about the number and height of line elevations. They were asked to answer the following question: "Do you think that you would pass under the line, in the present body orientation, imagining a virtual horizontal displacement of your body?".

A typical sequence of judgements happened as follows: the subjects were first tilted at the desired angle of orientation. This was followed by a $15 \mathrm{~s}$ period of rest, allowing the post-rotational effects issued from semi-circular canals stimulation to disappear (Benson 1990; Goldberg and Fernandez 1977). A loudspeaker, positioned in the 
subjects' median plane, $1 \mathrm{~m}$ behind the chair and elevated at eye level, emitted a first auditory signal indicating the trial onset, at which a luminous target appeared $(t=0 \mathrm{~s})$. Subjects had to keep their gaze on the target during all the visual presentation. At $t=2 \mathrm{~s}$, a luminous line appeared. Subjects were then required to orient their attention towards the luminous line projected in the peripheral field of vision and to estimate the possibility of passing under the line. At $t=6 \mathrm{~s}$, the luminous target and the line disappeared and a second auditory signal indicated it was time to respond via the push buttons ("able to pass" with the right hand-held button and "not able to pass" with the left hand-held button). The instructions were frequently repeated to keep subjects alert and concentrated on the task throughout the experiment. We assume that auditory signals did not affect visual localization, as no attentional focus on the spatial location of the sound was required (Bertelson and Radeau 1981; Pick et al. 1969; Warren 1979).

Eye movements were controlled online by means of a vertical EOG recording of the subjects' dominant eye. A consistent shift of the EOG signal indicated a change in gaze elevation whereas a sustained signal indicated a stabilization of gaze elevation. The signal polarity indicated the direction of vertical gaze displacement. Overall, subjects adequately performed the task. Nevertheless, if the DC signal indicated a change during the fixation task (e.g. a blink or an eye movement), the trial was immediately cancelled by the experimenter and presented again later in the session.

Finally, ten judgements (corresponding to ten line elevations randomly presented for different gaze elevations) were obtained within a sequence executed at the same body orientation. Each sequence ended by a rotation of the tilting chair back to the vertical and the room was lit for $15 \mathrm{~s}$ before a new sequence was launched. To limit the time spent on the experiment, a specific trial was presented only once for a total of 250 judgments. This design was chosen in accordance to the previous observations of Bringoux et al. (2008) who found high intrasubject judgment reliability after several trial repetitions in a similar perceptual task.

\section{Data processing}

Judgements were converted into binary values. A score of 1 was attributed when the subjects estimated they could pass under the line (in other words, when the line elevation was perceived higher than the minimal height for passing under). Conversely, a score of 0 was attributed when the subjects estimated they could not pass under the line. A Probit model, using a non-linear regression analysis for dichotomic variables, enabled us to determine the probability $P$ that subjects estimated at $50 \%$ that they could pass under the line. The Probit function was defined by the following relation:

$P_{i}=1 /\left(1+\left(C_{i, j} / C_{0}\right)^{n}\right)$

where " $P_{i}$ " is the probability that subjects estimated they can pass under the line. " $i$ " corresponds to the line number in the sequence, " $j$ " to the trial number, " $C_{0}$ " the line number for $P=0.5$ and " $n$ " the slope of the tangent at the inflection point of the curve. The latter coefficient constitutes an estimation of the discrimination sensibility relative to the chosen increments. An analysis of variance (ANOVA) with repeated measures was performed on " $n$ " values, to test any differences between the discrimination sensibility calculated for each experimental condition. Line elevations obtained at $P=0.5$ via the psychometric function defined judgements of subjective "passability", that is, estimates of the minimal height relative to eye level required for passing under obstacles. The estimates of subjective "passability", initially referred to eye-level for convenience, were subsequently reported to the top of the head, defined as the highest physical point of the head from the horizontal floor of the room measured for each subject in each body orientation. Hence, the data were expressed as a vertical elevation (in $\mathrm{cm}$ ) relative to the top of the head in order to define a true level of "passability". A repeated measures ANOVA was applied to the estimates of subjective "passability" relative to the top of the head in order to test any differences between body and gaze orientation angles and to investigate a possible interaction between the two factors. The influence of the egocentric position of the eyes was also investigated by a one way ANOVA applied to the estimates of subjective "passability" relative to eyein-head orientation. Finally, a multiple linear regression analysis was conducted on the mean estimates of subjective "passability" for each condition (i.e. for a specific body orientation associated to a particular gaze elevation) in order to investigate the presence of linear and independent effects of body and gaze orientation upon estimates and to characterize the magnitude of these effects.

\section{Results}

All the subjects stated that the required task was easy to perform and overall exhibited no hesitation when giving their response. Figure 2 illustrates the mean raw responses obtained for the different line elevations, relative to (a) body orientation and (b) gaze elevation. Overall, observation of the data showed that the higher the line relative to eye level, the more the subjects tended to answer that they could pass under, independently of their body orientation or their gaze elevation. Furthermore, these raw data also 


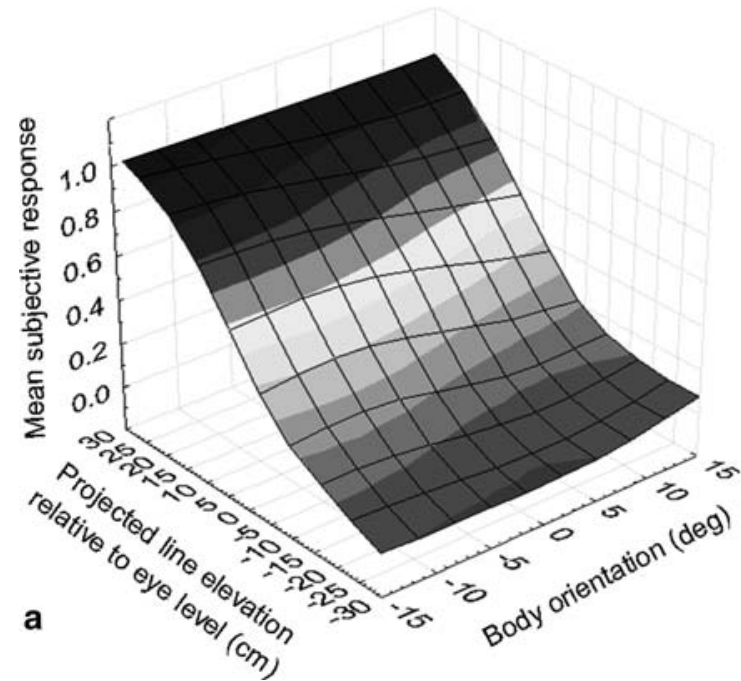

Fig. 2 Typical psychometric functions from all the subjects obtained via Probit non-linear regression analysis for a the different body orientations or $\mathbf{b}$ different gaze elevations. The mean subjective responses corresponded to the mean perceptual scores obtained when

suggested specific effects of body and gaze orientation upon estimates.

Probit analysis

A non-linear regression analysis (Probit function) was performed to determine the subjective "passability" for each subject in each experimental condition (see "Methods"). To assess the discrimination sensibility of the Probit processing, a five body orientations $\left(-10^{\circ} ;-5^{\circ} ; 0^{\circ} ;+5^{\circ} ;+10^{\circ}\right) \times$ five gaze elevations $\left(-10^{\circ} ;-5^{\circ} ; 0^{\circ} ;+5^{\circ} ;+10^{\circ}\right)$ repeated measures ANOVA was performed on the " $n$ " values (i.e. the slopes calculated at the inflection point of each function). Results showed there was no significant difference between body orientation angles $\left[F_{(4,44)}=1.75 ; P=0.16\right]$ or gaze elevation angles $\left[F_{(4,44)}=0.91 ; P=0.47\right]$. The interaction between both factors was also non-significant $\left[F_{(16,176)}=\right.$ $1.31 ; P=0.2]$. These results showed that the sensibility to discriminate the subjective "passability" for subjects did not differ between the experimental conditions.

\section{Mean comparisons of subjective "passability"}

The subjective "passability" relative to eye level obtained via the Probit analysis was reported to the true level of "passability", that is, relative to the top of the head for each subject at each body orientation angle. Overall, the subjective "passability" reported to the top of the head was found notably lower $(8.24 \mathrm{~cm})$ than the minimal physical height required for passing under the line. This denotes a significant over-estimation of the "passability" under obstacles.

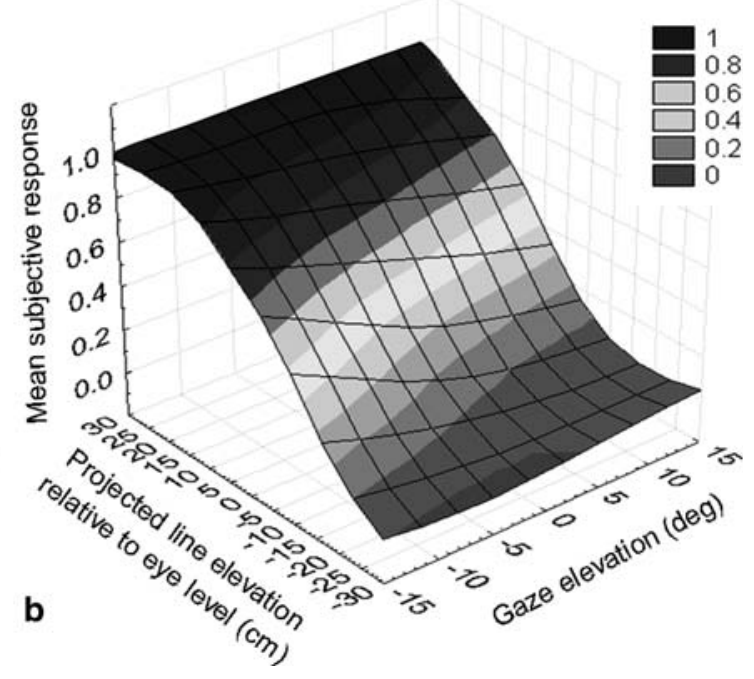

subjects had to estimate the minimal height for passing under. The value extracted at $P=0.5$ from each Probit function corresponds to the subjective "passability", that is, the perceived minimal height for passing under the line

A five body orientations $\left(-10^{\circ} ;-5^{\circ} ; 0^{\circ} ;+5^{\circ}\right.$; $\left.+10^{\circ}\right) \times$ five gaze elevations $\left(-10^{\circ} ;-5^{\circ} ; 0^{\circ} ;+5^{\circ} ;+10^{\circ}\right)$ ANOVA conducted on the mean estimates of subjective "passability" revealed a significant effect of body orientation $\left[F_{(4,44)}=7.5636 ; P<0.001\right]$ and gaze elevation $\left[F_{(4,44)}=9.5481 ; P<0.001\right]$ on the estimated possibility of passing under the line. The interaction between both factors was not significant $\left[F_{(16,176)}=0.74 ; P=0.75\right]$. This means that the main effect of body orientation was not affected by gaze elevation and vice versa. Post hoc analyses (Newman-Keuls test) showed significant differences between body orientation angles (Fig. 3).

A nine eye-in-head orientations $\left(-20^{\circ} ;-15^{\circ} ;-10^{\circ}\right.$; $-5^{\circ} ; 0^{\circ} ;+5^{\circ} ;+10^{\circ} ;+15^{\circ} ;+20^{\circ}$ ) ANOVA conducted on the mean estimates of subjective "passability" showed non-significant differences between eye-in-head orientation conditions $\left[F_{(8,88)}=1.21 ; P=0.30\right]$. Then, the judgement of subjective "possibility" was not affected by the egocentric position of the eyes relative to the head.

\section{Multiple linear regression analysis}

A multiple regression analysis, applied to the mean estimates of subjective "passability" obtained for all the subjects in all experimental conditions, showed a linear effect of body orientation and an independent linear effect of gaze elevation on the estimated possibility of passing under high obstacles $\left[F_{(2,22)}=81.84 ; P<0.001\right]$. Figure 4 shows that most of the data fit on a simple plane when plotted as a function of body and gaze orientation.

These results showed that the error on estimating the possibility of passing under high obstacles is both 


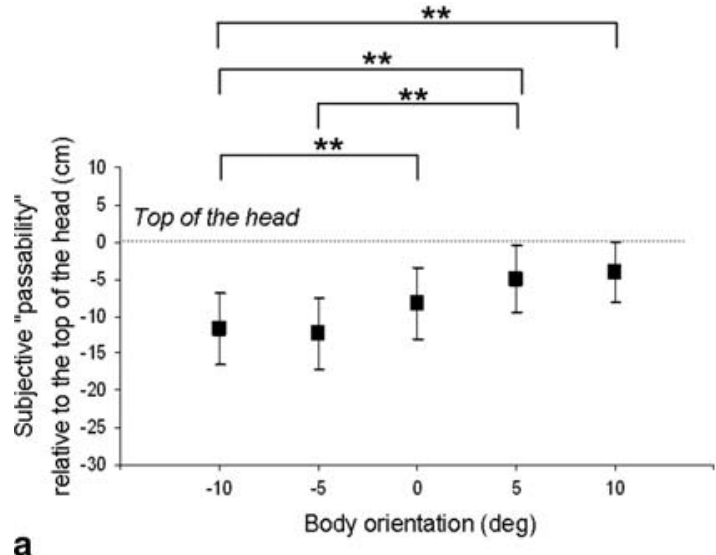

Fig. 3 Mean subjective "passability" relative to the top of the head (plotted with \pm confidence intervals) obtained for a the different body orientations or $\mathbf{b}$ for the different gaze elevations. The zero corresponds to the top of the head reference (i.e. the highest point of

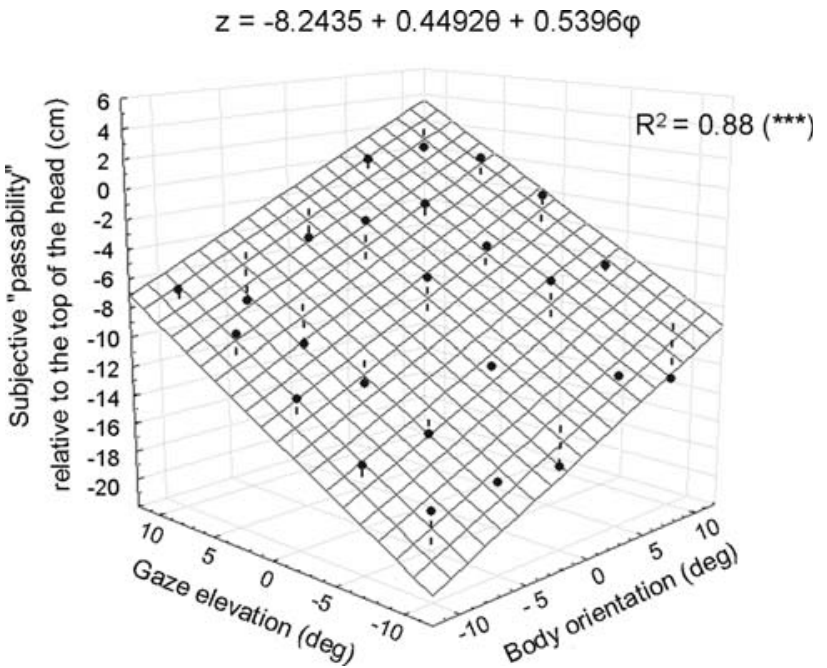

Fig. 4 Multiple regression function fitted to the mean estimates of subjective "passability". Mean estimates of subjective "passability" relative to the top of the head (black circles) are plotted against body orientation and gaze elevation. The hatched area represents the multiple regression plane, whose regression equation is given above the graph: " $z$ " corresponds to the subjective "passability" relative to the top of the head, " $\theta$ " to the body orientation angle, and " $\varphi$ " to the gaze elevation angle. The length of the segments joining the black circles to the plane represents the deviation of the subjective "passability" from the plane. The $R^{2}$ indicates the significance level of the fit

proportional to the body orientation angle and to the gaze elevation angle. They also showed that the independent effects of body and gaze orientation combined additively. The multiple linear regression analysis is characterized by the following function:

$z=0.45 \theta+0.54 \varphi-8.24$

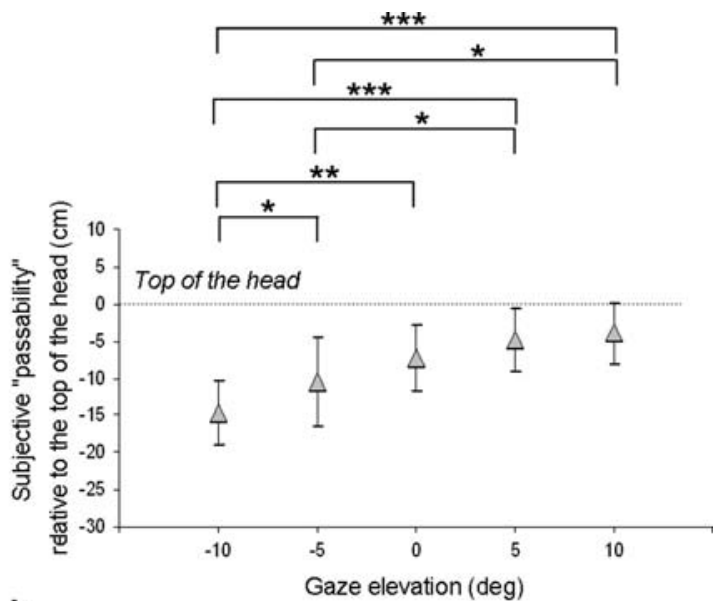

b

the head irrespective of head orientation). Significant differences between body and gaze orientation angles (Newman-Keuls test) are also shown $(* * * P<0.001 ; * * P<0.01 ; * P<0.05)$

where " $z$ " corresponds to the subjective "passability" relative to the top of the head, " $\theta$ " to the body orientation angle, " $\varphi$ " to the gaze elevation angle. The coefficient associated to the weight of the body orientation influence is $0.45 ; 0.54$ is the coefficient associated to the weight of the gaze orientation influence and -8.24 corresponds to the mean calculated subjective "passability" relative to the top of the head.

\section{Discussion}

The main purpose of this study was to investigate the influence of gaze elevation on estimating the possibility of passing under high obstacles during whole-body tilt, while stationary, and in absence of visual allocentric cues. Specifically, the question was to determine whether gaze elevation could constitute an egocentric influence which may in turn affect geocentric estimates. The second objective of this work was to question the relation between body orientation and gaze elevation on these perceptual judgements.

Overall, our results showed that the mean subjective "passability" is $-8.24 \mathrm{~cm}$ (i.e. $-2.07^{\circ}$ ) lower than the physical minimal height required to adequately perform the task. In other words, subjects estimated they were able to pass under obstacles which were actually located below the top of their head. These results highlighted a global overestimation of the possibility of passing under obstacles. Recently, Bringoux et al. (2008), using the same experimental setup as the one designed in the present study, found a similar overestimation of the subjective "passability" during body tilt. This result has been related to the 
perceived visual horizon, which was found globally lower than the physical reference in darkness (Bringoux et al., 2008). For instance, the measured offset was $-3^{\circ}$ in the same experimental setup. Although the subjective visual horizon was not recorded in the present experiment, the occurrence of such a phenomenon is clearly assumed in this study. Other previous works also reported that the subjective visual horizon is lower in darkness (Bringoux et al. 2004, 2008; MacDougall 1903; Raphel and Barraud 1994; Sharp 1934; Stoper and Cohen 1986). This phenomenon may be related to the $30^{\circ}$ backward orientation of the saccular and utricular maculae relative to the head (Rosenhal 1972; Bortolami et al. 2006). As a consequence, obstacles, whose elevation is referred to the subjective visual horizon, will be considered higher than they actually are, since the visual horizon is perceived lower than its true location in darkness.

Gaze elevation effect on geocentric judgments

Our results showed a significant effect of gaze elevation on estimating the possibility of passing under high obstacles. Specifically, the more the gaze was orientated downward, the more the possibility of passing under high obstacles was overestimated. Conversely, this overestimation was reduced when the gaze was orientated upward. According to many studies, gaze constitutes an egocentric reference that may be advanced as a potential source of egocentric attraction reported on geocentric judgments.

Numerous authors have stressed the importance of eye level in height and distance judgments (Li et al. 2001; Matin and Li 1995; Ooi et al. 2001). Specifically, eye level is commonly considered as a central reference in egocentric (Matin and Li 1995) and geocentric spatial localization in darkness (Bringoux et al. 2004, 2008; Stoper and Cohen 1989). In parallel, Poljac and van den Berg (2005) and Poljac et al. (2005) have investigated the importance of the plane of regard in egocentric spatial localization. In a first study, subjects were asked to point with their supported arm to their plane of regard (Poljac and van den Berg 2005). The results showed a correct localization of this plane in space. In a second study, subjects were asked to perceptually estimate the elevation of flashed probe points relative to their plane of regard during eccentric viewing (Poljac et al. 2005). These results showed that the elevation of objects relative to this plane was perceived accurately, irrespective of eye or head orientation. These findings suggest that passive object localization relative to an egocentric reference is correctly achieved along the vertical dimension, contrary to what has been reported for judgements of object lateral eccentricity in the peripheral field assessed via pointing movements (Bock 1993). In the latter case, the necessary transformation of sensory coordinates into an appropriate motor output could explain the errors reported in pointing judgments (McIntyre et al. 1997).

In line with the conclusion of Poljac et al. (2005), stating that "the plane of regard is a good starting point for representing objects in head-centric coordinates", our results demonstrated that the plane of regard is also involved in judging the location of objects with respect to a geocentric reference frame (i.e. including the horizontal plane passing through the eyes). The linear effect of gaze elevation on estimating the possibility of passing under high obstacles observed in our study implies that gaze elevation influence is not magnified for the maximal gaze elevation angles tested in this experiment. In other words, gaze elevation exerted a linear egocentric attraction upon geocentric judgments in a range from $-10^{\circ}$ to $+10^{\circ}$ (a range limited by some morphological constrains at extreme body tilts such as the curvature of the brow).

Additive independent effects of body and gaze orientation

The results did not reveal any interaction between body and gaze orientation. Specifically, in the range of the tested orientations, body orientation effect is not influenced by gaze elevation angle, and conversely, gaze elevation effect is not affected by body orientation angle. Additive effects of body and gaze orientation were also shown. Moreover, our results showed that several egocentric references may have additive effects and participate, each independently, in the construction of a resultant egocentric influence upon geocentric judgments.

Some previous studies suggested that multiple body parts could constitute egocentric references which additively combine to yield main effects on geocentric judgments. For instance, Guerraz et al. (1998) showed that lateral head tilt alone and lateral trunk tilt alone generated single effects on subjective visual vertical estimates, which could merge into a cumulative main egocentric effect when head and trunk are tilted together. Moreover, some authors (Becker et al. 2000; Ito and Gresty 1997) suggested that multiple body parts could be involved in the elaboration of the idiotropic vector influencing verticality perception. Consequently, one might expect that the whole-body configuration in space (sitting or upright posture) could modify the perception of some geocentric directions of space involved in the judgement of "passability" under obstacles.

Interpenetrability between reference frames

The extent to which reference frames are implicated in spatial cognition tasks is still widely discussed. Several hypotheses have been proposed in the literature. 
The first hypothesis suggests that subjects can rapidly adapt their behaviour by switching from a specific reference frame to another while performing their task. For instance, Ghafouri et al. (2002) identified a radical switch between allocentric and egocentric references frames during fast arm pointing movements. In this task, subjects had to point either to a motionless target or to a target moving synchronously with the trunk. In this context, reference frames could be considered as pre-existing neurophysiological structures, some exclusive from others (Galati et al. 2000).

The second hypothesis supports the existence of intermediate states, in which egocentric, allocentric and geocentric cues would merge into a hybrid reference frame (Flanders and Soechting 1995; Kappers 2003, 2004; Paillard 1991; Soechting and Flanders 1992). For instance, Kappers (2004) found a combined contribution of allocentric and egocentric cues in the haptic judgment of parallelism. Blindfolded subjects exhibited systematic deviations when manually rotating a test bar in such a way that they felt it as parallel relative to a reference bar in the midsagittal plane. In the same vein, Coello and Iwanow (2006) found an influence of allocentric cues (given by a structured background) on an egocentric pointing task (i.e. pointing movements towards a visual target located at various distances along the sagittal axis). Finally, according to Bringoux et al. (2004, 2007, 2008), the present findings illustrate the "interpenetrability" between reference frames.

Two cases of "interpenetrability" have been described in the literature. The first relates to the existence of a dynamic intermediate state, where the weight attributed to each reference frame evolved during the task. Specifically, this phenomenon has been observed on the rod-and-frame effect during head tilt (DiLorenzo and Rock 1982) or whole-body tilt (Bishof 1974; Goodenough et al. 1985; Zoccolotti et al. 1992). For instance, it was shown that a $45^{\circ}$ head tilt increased the influence of a $20^{\circ}$ tilted frame upon visual vertical estimates, compared to a head upright condition (DiLorenzo and Rock 1982). The greater rodand-frame effect was explained by the decreased efficiency of available gravity cues during head tilt, but might also be understood as an increased weight of the allocentric (visual) frame of reference when the head is no longer aligned with gravity.

The second case refers to the existence of an intermediate reference frame in which the contribution of each egocentric, allocentric or geocentric cues is kept constant and stable throughout the task (Bringoux et al. 2004, 2007, 2008; Kappers 2003, 2004; Neggers et al. 2005). For instance, Bringoux et al. (2008), showed that egocentric references could influence the perceived location of objects relative to some geocentric references, each with a constant weight, whatever the tilt magnitude. In the same perspective, Neggers et al. (2005) showed that allocentric cues, given by a structured visual background placed behind a target, biased judgements of the target's location relative to the body with a constant weight.

According to the latter hypothesis, our study strongly suggests that perceptual shifts in judging the "passability" under obstacles may result from the "interpenetrability" between egocentric and geocentric reference frames. This finding might lead to a new and hybrid reference frame, corresponding to a sustained intermediate state between a geocentric reference frame normally required to adequately perform the task, and a disturbing egocentric reference frame. Successive transformations of coordinates required to perform the task (Matin and Li 1992; Stoper and Cohen 1989) may account for the influence of one reference frame to another.

\section{Conclusion}

To our knowledge, the present study is the first to show an independent, linear influence of gaze elevation on estimating the possibility of passing under high obstacles, while stationary and in the absence of allocentric cues. Furthermore, our results suggest that gaze elevation is additively combined to body orientation to yield a resultant egocentric effect that modifies geocentric estimates. The present work also supports the hypothesis of "interpenetrability" between egocentric and geocentric reference frames to explain how judgements of "passability" under obstacles are attracted towards body orientation and gaze elevation. Further experiments investigating the orientation of different body segments in space should be particularly interesting to better understand the egocentric influences upon judgements of subjective "passability" under obstacles.

Finally, our study may have important repercussions in aeronautics where pilots are usually seated $30^{\circ}$ backward (Roumes and Grau 2003). Comparable egocentric attraction upon spatial estimates may arise when pilots have to elevate their gaze towards a vertical visual display, while controlling the pitch of their aircraft. Mars et al. (2004, 2005) showed that head and body orientation are of importance in judging the pitch of aircrafts. Our study suggests that gaze elevation should also be taken into account in the conception of visual displays to prevent pilots from risks of spatial disorientation, specifically under visually poor flight conditions (Braithwaite et al. 1998; Kirkham et al. 1978).

In addition, because gaze orientation and attentional focus are often congruent in everyday life, further experiments dissociating gaze orientation and attentional location 
might also be investigated to reduce the risks of accidents in aeronautics. Specifically, in accordance with the paradigm of Posner et al. (1980), it could be valuable to determine whether priming cues orienting attention would affect geocentric estimates in the same way as gaze elevation.

Acknowledgments Aurore Bourrelly was supported by a grant from DGA-CNRS (No. 2007-746). The authors are grateful to Gabriel Gauthier, Franck Buloup, and Alain Donneaud for their technical expertise and Cecile Scotto for her help during data acquisition and processing. They also thank Thelma Coyle, Julie Martin-Malivel and George Mitchell for English corrections, and the anonymous reviewers for their helpful comments in revising the current manuscript.

\section{References}

Becker W, Jürgens R, Boß T (2000) Vestibular perception of selfrotation in different postures: a comparison between sitting and standing subjects. Exp Brain Res 131(4):468-476

Benson AJ (1990) Sensory functions and limitations of the vestibular system. In: Warren R, Wertheim AH (eds) Perception and control of self-motion. Erlbaum, Hillsdale, pp 145-170

Bertelson P, Radeau M (1981) Cross-modal bias and perceptual fusion with auditory-visual discordance. Percept Psychophys 29(6):578-584

Bishof N (1974) Optic-vestibular orientation to the vertical. In: Kornhuber HH (ed) Handbook of sensory physiology, vestibular system part 2: psychophysics, applied aspects and general interpretations, vol 6. Springer, New York, pp 155-190

Bock O (1993) Localization of objects in the peripheral visual field. Behav Brain Res 56:77-84

Bortolami SB, Pierobon A, DiZio P, Lackner JR (2006) Localization of the subjective vertical during roll, pitch, and recumbent yaw body tilt. Exp Brain Res 173(3):364-373

Braithwaite MG, Douglass PK, Durnford SJ, Lucas G (1998) The hazard of spatial disorientation during helicopter flight using night vision devices. Aviat Space Environ Med 69(11):10381044

Bringoux L, Tamura K, Faldon M, Gresty MA, Bronstein AM (2004) Influence of whole-body pitch tilt and kinesthetic cues on the perceived gravity-referenced eye level. Exp Brain Res 155(3): 385-392

Bringoux L, Mezey LE, Faldon M, Gresty MA, Bronstein AM (2007) Influence of pitch tilts on the perception of gravity-referenced eye level in labyrinthine defective subjects. Neuropsychologia 45(2):350-356

Bringoux L, Robic G, Gauthier GM, Vercher JL (2008) Judging beforehand the possibility of passing under obstacles without motion: the influence of egocentric and geocentric frames of reference. Exp Brain Res 185(4):673-680

Coello Y, Iwanow O (2006) Effect of structuring the workspace on cognitive and sensorimotor distance estimation: no dissociation between perception and action. Percept Psychophys 68(2):278-289

DiLorenzo JR, Rock I (1982) The rod-and-frame effect as a function of the righting of the frame. J Exp Psychol Hum Percept Perform 8(4):536-546

Flanders M, Soechting JF (1995) Frames of reference for hand orientation. J Cogn Neurosci 7(2):182-195

Galati G, Lobel E, Vallard G, Berthoz A, Pizzamiglio L, Le Bihan D (2000) The neural basis of egocentric and allocentric coding of space in human: a functional magnetic resonance study. Exp Brain Res 133(2):156-164
Ghafouri M, Archambault PS, Adamovich SV, Feldman AG (2002) Pointing movements may be produced in different frames of reference depending on the task demand. Brain Res 929(1): $117-128$

Gibson JJ (1979) The ecological approach to visual perception. Houghton Mifflin, Boston

Goldberg JM, Fernandez C (1977) Conduction times and background discharge of vestibular afferents. Brain Res 122(3):545-550

Goodenough DR, Cox PW, Sigman E, Strawderman WE (1985) A cognitive-style conception of the field-dependence dimension. Cah Psychol Cogn 5:687-706

Guerraz M, Poquin D, Luyat M, Ohlmann T (1998) Head orientation involvement in assessment of the subjective vertical during whole body tilt. Percept Mot Skills 87(2):643-648

Howard IP (1982) Human visual orientation. Willey, New York

Ito Y, Gresty MA (1997) Subjective postural orientation and visual vertical during slow pitch tilt for the seated human subject. Aviat Space Environ Med 68(1):3-12

Kappers AML (2003) Large systematic deviations in a bimanual parallelity task: further analysis of contributing factors. Acta Psychol 114(2):131-145

Kappers AML (2004) The contributions of egocentric and allocentric reference frames in haptic spatial tasks. Acta Psychol 117(3): 333-340

Kirkham WR, Collins WE, Grape PM, Simpson JM, Wallace TF (1978) Spatial disorientation in general aviation accidents. Aviat Space Environ Med 49(9):1080-1086

Li W, Dallal N, Matin L (2001) Influences of visual pitch and visual yaw on visually perceived eye level (VPEL) and straight ahead (VPSA) for erect and rolled-to-horizontal observers. Vision Res 41(22):2873-2894

MacDougall R (1903) The subjective horizon. Psychol Rev 4: $145-166$

Marcilly R, Luyat M (2008) The role of eye height in judgment of an affordance of passage under a barrier. Curr Psychol Lett 24(1):12-24

Mark LS (1987) Eyeheight-scaled information about affordances: a study of sitting and stair climbing. J Exp Psychol Hum Percept Perform 13(3):361-370

Mars F, Vercher JL, Blouin J (2004) Perception of the vertical with a head-mounted visual frame during head tilt. Ergonomics 47:1116-1130

Mars F, Bringoux L, Cian C, Barraud PA, Raphel C, Vercher JL (2005) Perception de la verticale avec un cadre visuel solidaire de la tête : implications pour l'utilisation des visiocasques. Trav Hum 68:125-152

Matin L, Li W (1992) Visually perceived eye level: changes induced by a pitched from-vertical 2-line visual field. J Exp Psychol Hum Percept Perform 18(1):257-289

Matin L, Li W (1995) Multimodal basis for egocentric spatial localization and orientation. J Vestib Res 5(6):499-518

McIntyre J, Stratta F, Lacquaniti F (1997) Viewer-centered frame of reference for pointing to memorized targets in three-dimensional space. J Neurophysiol 78(3):1601-1618

Mittelstaedt H (1983) A new solution to the problem of the subjective vertical. Naturwissenschaften 70(6):272-281

Mittelstaedt H (1986) The subjective vertical as a function of visual and extraretinal cues. Acta Psychol 63:63-85

Neggers SFW, Schölvinck ML, van der Lubbe RHJ, Postma A (2005) Quantifying the interactions between allo- and egocentric representations of space. Acta Psychol (Amst) 118(1-2):25-45

Ooi TL, Wu B, He ZJ (2001) Distance determined by the angular declination below the horizon. Nature 414:197-200

Paillard J (1991) Motor and representational framing of space. In: Paillard J (ed) Brain and space. Oxford University Press, Oxford, pp 163-182 
Pick HL, Warren DH, Hay JC (1969) Sensory conflict in judgment of spatial direction. Percept Psychophys 6:203-205

Poljac E, Lankheet MJM, van den Berg AV (2005) Perceptual compensation for eye torsion. Vision Res 45(4):485-496

Poljac E, van den Berg AV (2005) Localization of the plane of regard in space. Exp Brain Res 163(4):457-467

Posner MI, Snyder CRR, Davidson BJ (1980) Attention and detection of signals. J Exp Psychol Gen 102(2):160-174

Raphel C, Barraud PA (1994) Perceptual thresholds of radial accelerations as indicated by visually perceived eye level. Aviat Space 65:204-208

Roumes C, Grau JY (2003) Rafale: a human factors view of the man machine interface. Med Aeronaut Spat 45:23-26

Rosenhal U (1972) Vestibular macular mapping in man. Ann Otol Rhnol Laryngol 81:339-351

Sharp WL (1934) An experimental study concerning visual localisation in the horizontal plane. J Exp Psychol (Hum Percept) 17:787-797

Soechting JF, Flanders M (1992) Moving in three-dimensional space: frames of reference, vectors, and coordinate systems. Annu Rev Neurosci 15:167-191
Stoper AE, Cohen MM (1986) Judgements of eye level in light and in darkness. Percept Psychophys 40:311-316

Stoper AE, Cohen MM (1989) Effect of structured visual environments on apparent eye level. Percept Psychophys 46(5):469-475

Van der Meer ALH (1997) Visual guidance of passing under a barrier. Early Dev Parent 6:149-157

Wagman JB, Malek EA (2008) Perception of whether an object affords walking under from different points of observation. Ecol Psychol 20:65-83

Warren DH (1979) Spatial localization under conflict conditions: is there a single explanation? Perception 8:323-337

Warren WH Jr (1984) Perceiving affordances: visual guidance of stair climbing. J Exp Psychol Hum Percept Perform 10(5):683-703

Warren WH Jr, Whang S (1987) Visual guidance of walking through apertures: body-scaled information for affordances. J Exp Psychol Hum Percept Perform 13(3):371-383

Zoccolotti P, Antonucci G, Goodenough DR, Pizzamiglio L, Spinelli D (1992) The role of frame size on vertical and horizontal observers in the rod-and-frame illusion. Acta Psychol 9(2): 171-187 